\title{
A case for a science-informed perspective on health care reform
}

A s a physician-scientist elected to represent the American Society for Clinical Investigation, I hope to provide a voice for at least some of the physician-scientists who tirelessly devote their energies to translating their findings from the laboratory to improve patient care. We labor because we believe elucidating the molecular underpinnings of disease will translate into more effective treatments that ease the burden of disease suffering and improve the lives of millions of Americans (and people across the globe). That is what drives us. Many of us also believe that molecular medicine, by directing more specific, less toxic treatments to subsets of patients most likely to benefit from them, can also reduce medical costs and improve care. Thus, we are deeply invested in the outcome of the health care debate. As an individual, I hope for health care reform that is humane and pervasive, universal in scope, and science-informed in its details - in short, the kind of health care reform our country must have to be the civilized beacon of hope it deserves to be.

Through application of powerful technologies to study genes, proteins, and metabolites in human patients, we have learned that a variety of different molecular alterations can underlie phenotypically similar diseases. An entity originally considered as one homogeneous disease more likely represents multiple pathogenic routes toward a common disease phenotype. This distinction is more than academic when drugs that target specific genetic or biochemical altera- tions are used as treatments. The average American does not need to understand molecular biology to recognize that their health care could be greatly affected by this. Take cancer as an example. Virtually every American will be challenged by cancer, either their own or in a loved one. We are all likely to become familiar at some point in our lives with the pain and disappointment of traditional anticancer treatments, including cytotoxic chemotherapy and radiation, that are toxic and only modestly improve the lifespan for most patients whose cancers are detected at an advanced stage. Molecular medicine promises to change this.

Studying the underpinnings of cancer has begun to yield new treatments that target specific molecular lesions needed by the cancer cells, providing the potential for much more effective, less toxic treatments. These targetable lesions are usually only present in a subset of patients with that cancer type. In fact, most patients with that type of cancer won't benefit. Traditional metrics would put these new targeted treatments into the "doesn't work" category, yet for patients with the specific targetable lesion, these treatments may be life saving. One can imagine a future in which each patient's cancer is analyzed for targetable lesions and then a personalized treatment plan is tailored to best treat their disease. Thus, all Americans have a stake in the outcome. A path toward realizing this future will require forward-thinking, scienceinformed health care reform that develops, tests, and incorporates biomarkers into the determination of efficacy of new treatments in well-designed clinical trials so that we can better answer the question "What works and what doesn't?" by asking, "What works for whom?" I believe that if most Americans were to recognize this fact, they would gladly invest in a process to personalize their care.

I am not an economist. I claim no expertise in understanding the full scope of budgetary issues shaping this challenge. However, I believe that this is a debate about more than just cutting costs and improving access to care. It is also an opportunity to develop a framework for evaluating new tests and treatments that are being dramatically transformed by advances in molecular medicine. As our elected leaders craft policy that will determine whether America will be a model for the seamless integration of cutting-edge science into a health care delivery system that is effective and sustainable, I hope they will: (a) recognize the impact of disease heterogeneity on evaluation of new diagnostics and treatment strategies; (b) encourage development of biomarkers to guide treatment toward patients most likely to benefit; and (c) provide support for well-designed clinical trials to evaluate their efficacy.

\section{Paul Mischel}

David Geffen School of Medicine at UCLA, Los Angeles, California, USA. E-mail:pmischel@ mednet.ucla.edu.

J. Clin. Invest. 119:2855 (2009). doi:10.1172/ JCI41038.

\section{Creating meaningful health care reform}

1. n the heated debate about health care reform, there has been little serious discussion about how to fix the extraordinarily expensive and inefficient delivery system that makes meaningful expansion of coverage difficult. The public debate has degenerated into polemics, while the real issue of providing better health care is lost in the fray. The know-how and capability to create a far more rational and cost-effective system is within our grasp, and there has never been a more important time for the medical profession to take the lead in advocating for reform. Academic physicians are highly respected by the public and politicians; our views are valued, and we must engage more actively to support better approaches to health care. To improve care, I believe that practice must shift from a reactive, sporadic, disease event-oriented approach to one that promotes health, prevents disease, and intervenes early and effectively when it occurs. To do this, we must combine three key elements: (a) a personalized strategic approach to care with meaningful patient engagement, (b) a delivery system designed to support and coordinate care over time, and (c) a rational reimbursement system. 
The current approach unfortunately contains none of these elements, but the resources to do so are available. We are experiencing a revolution in our ability to predict disease, track its progress, and intervene coherently. These new capacities, stemming in part from genomics, systems biology, and advanced medical technologies, can improve care by making it personalized, predictive, preventive, and costeffective. Capabilities are being improved to define each individual's risk for developing chronic diseases as well as to detect the onset and provide the best personalized treatment when needed. We already have the know-how to create strategic solutions to improving health and minimizing disease (1). Such approaches, called prospective care, personalized medicine, integrative care, or patient-centered care, provide individuals with an assessment of their health risks and the education and support needed to create their strategic health plan. This is facilitated by an ongoing relationship with a health system that provides a primary point of contact; i.e., primary care physician and/or health care coach and coordinated care over time. These capabilities can be far more cost-effective, as they heighten the involvement and compliance of the individual and organize care over time. In contrast, the system today focuses on poorly coordinated and expensive treatment of episodes of late-stage chronic disease, many of which are preventable.

As a physician with 40 years' experience in virtually all aspects of medicine, I believe that our profession has an obligation to provide a strong voice in the health care debate. My belief is that the needed outcomes for a rational approach to care include:

Accessibility. Health is among our greatest resources and a basic value. Access and insurance to support health and minimize and treat disease should be available for all Americans.

Education. Educating the public about the importance of health and one's ability to influence it should be a major societal focus. Why not advocate a broad "going healthy" movement similar to the "going green" initiative to focus our nation on improving health as a national goal and a shared responsibility? Businesses, schools, communities, consumer groups, social and religious organizations, public health groups, and the government could develop collaborations focusing on improving our nation's health.

Approach to care. Strategic health planning based on a patient's risks for preventable diseases along with appropriate tracking and early personalized intervention are crucial. Chronic diseases develop over time, so there are abundant opportunities for personalization, prevention, and minimization of disease progression. The basic medical work-up should move from its current "find it and fix it" mind-set to one that is also proactive and preventive.

Delivery systems. Health providers should be organized to give the patient ongoing and coherent care at the level needed, from health promotion to treatment of disease events.

Biomedical research and continuous improvement. Prediction, prevention, and personalization are hallmarks of rational health care. These capabilities require constant advancement, and thus support is needed for robust biomedical research and translation. Regulatory processes should be created to allow rapid adoption of validated discoveries.

Reimbursement. Strategies to pay for prevention, continuity of care, and better outcomes are essential. Our current system rewards interventions for disease events and thus encourages the care we have today. Reimbursement for prevention, early intervention, and effective long-term management is missing. Of all the impediments to reform, changes in reimbursement may be the most difficult to overcome, as there is so much money at stake. Nonetheless, reimbursing pro- viders for better clinical outcomes will have the greatest leverage in fostering better models of care.

Creating and validating better care models. While the concepts of personalized, prospective care are sound and indications of effectiveness apparent, we must develop more working models and learn from those that work the best. To stimulate competition and ingenuity, demonstration projects funded by the government and private insurers should be established. Outcomes could be monitored and overseen by credible, nonbiased entities such as the Institute of Medicine.

I believe that as a nation, we must affirm our commitment to a reasonable and practical level of health care as an individual's right. Health care must be directed toward enhancing patient involvement to improve their health along with systems to support this. Personalized, predictive, preventive, and coordinated strategies can replace the current approach, which wastefully focuses on disease events. I consider our health care system to be the best in the world in dealing with disease events, yet sorely inadequate in preventing and minimizing disease - we can and we must do better. Resolving this dilemma is at the crux of meaningful health care reform, and, as physicians, we must provide a voice of reason in this debate.

\section{Ralph Snyderman}

Duke University Medical Center, Durham, North Carolina, USA. E-mail: ralph. snyderman@duke.edu.

Conflict of interest: Ralph Snyderman is chairman of Proventys Inc.

J. Clin. Invest. 119:2855-2856 (2009). doi:10.1172/JCI41034.

1. Snyderman, R. 2004. The AAP and the transformation of medicine. J. Clin. Invest. 114:1169-1173.

\section{Health care reform - need for less emotion and more science}

he United States is currently engaged in a very important debate on the issue of health care reform. There is much rhetoric about the dangers of reform, the negative role of government versus the private sector in administering health care, the risk of losing personal control over important health care choices, the merits and weaknesses of a single-payer system, the value of 